\title{
LA TRADUCCIÓN Y COMUNICACIÓN DEL CONSENTIMIENTO INFORMADO COMO MEDIDA PARA GARANTIZAR SU COMPRENSIBILIDAD
}

\author{
Elena Pérez Estevan \\ Universidad de Alcalá \\ elena.perez@uah.es
}

\section{Resumen}

En esta era de globalización y migración, el modelo sanitario paternalista se ve afectado por un nuevo rumbo donde el paciente también adquiere protagonismo y, por ende, un papel activo. La diversidad de pacientes que acuden cada día a los centros sanitarios españoles comporta distintos escenarios comunicativos para los que la figura del traductor e intérprete médico ocupa un papel fundamental. Uno de estos escenarios es la entrega de documentos dirigidos a pacientes en la consulta médica con pacientes extranjeros. El presente trabajo revisa las líneas de investigación del consentimiento informado desde la legibilidad y la comprensibilidad. Se presenta un estudio cualitativo y cuantitativo sobre la percepción de los odontólogos profesionales del papel del intérprete y las necesidades de una muestra de pacientes autóctonos y extranjeros como receptores.

\begin{abstract}
"Informed consent translation and communication as a tool to ensure its comprehensibility"

In the globalization and migration era, the paternalistic model in healthcare is affected by a new direction giving way to patient prominence and, therefore, activation. The diversity of patients in the Spanish medical consultations involves different communicative scenarios in which the medical interpreter and translator plays a crucial role as for example the delivery of documents addressed to the patient in the medical consultation with foreign patients. In this paper, we integrate research in informed consent from readability and comprehensibility. A qualitative and quantitative study
\end{abstract}


on how professional dentists perceive the interpreters' role is carried out. The needs of a sample of native and foreign patients as users are also covered.

Palabras clave: Consentimiento informado. Papel del intérprete. Comunicación médico-sanitaria. Empoderamiento del paciente. Comprensibilidad.

Keywords: Informed consent. Interpreters' role. Medical communication. Patient empowerment. Comprehensibility. 


\section{Un cambio de paradigma: del paternalismo sanitario al empoderamiento del paciente}

En los últimos años hemos sido testigos del inicio de un cambio en el modelo de asistencia sanitaria de los países occidentales desarrollados a causa de la ampliación y el acceso de las vías del conocimiento y la tendencia a la instrucción del paciente (Epstein et al. 2005: 1520; Mayor Serrano 2005: 133; Muñoz Miquel 2014: 172). Este cambio en la relación entre el profesional sanitario y el paciente conlleva una necesaria creación de materiales adaptados o que incluyan al paciente. En este punto es donde los traductores, intérpretes, mediadores y redactores médicos pueden realizar aportaciones y tienen un amplio campo de actuación para adaptar los mencionados materiales en términos de bagaje cultural, situación, enfermedad y necesidades, entre otros. Para divulgar el conocimiento especializado, se han contemplado y estudiado distintas vertientes de la traducción como la traducción heterofuncional, consistente en la reformulación de la información para que el texto meta tenga una función distinta a la del texto origen (García Izquierdo \& Montalt i Resurrecció 2013: 47).

En la mayoría de los documentos que se redactan no se tiene en cuenta a los distintos lectores a los que puede llegar este tipo de documentación; es decir, se dirigen a un paciente estándar. Por tanto, si cada vez más los profesionales sanitarios aúnan sus esfuerzos para personalizar la medicina (Sánchez Martos 2014: 96-104), ¿por qué no personalizar la comunicación a través de la información escrita y oral? ¿Quién debe iniciar dicho proceso?

\section{Comunicación médico-sanitaria y géneros textuales}

\subsection{La importancia de la figura del traductor/redactor o intérprete médico}

Ante el cambio de modelo de atención sanitaria, los pacientes quieren y necesitan un mayor acceso a la información escrita y oral para adoptar un papel más activo en su toma de decisiones. La problemática viene dada por el vacío en formación relativa a la comunicación de los profesionales que no disponen o no se les ha entrenado para divulgar el conocimiento especializado, como ya comentaron García Izquierdo \& Montalt i Resurrecció (2013). 
Dentro de la interpretación sanitaria, textos como los consentimientos informados exponen al intérprete a un desafío multidisciplinar para lograr una comunicación eficaz. No solamente se requiere del conocimiento de la terminología específica en áreas temáticas afines, sino que es fundamental su familiarización con los principios, el propósito y las distintas formas de presentación de dicho material, el trabajo en equipo con el profesional sanitario, etc.

Por ello, es de suma importancia tratar de esclarecer qué papel adopta el intérprete en el proceso del consentimiento informado. Recordemos que partimos de un documento escrito que requiere de interacción oral.

En la última década son muchos los estudios encaminados a esclarecer o categorizar el papel del intérprete en el ámbito sanitario. Destacarían modelos como el de abogacía e imparcialidad propuesto por Cambridge (2002) o el enfoque de Leanza (2005) sobre el papel del intérprete como facilitador de la comunicación o asimilador cultural y, en la última década, el modelo patient navigators propuesto por Crezee (2013). Este último modelo hace hincapié en las diferencias culturales que pueden darse en la consulta y es el intérprete el que interviene a modo de enlace cultural, como expresa la propia autora (Crezee 2013: 17):

Even if the interpreter interprets such words appropriately, a culture-specific misunderstanding may arise and the interpreter may need to act as a cultural liaison and resolve the resulting communication breakdown.

\subsection{Comunicación orientada hacia el usuario}

En el contexto sanitario, una correcta comunicación es fundamental e indispensable, aunque en algunos contextos pueda verse infravalorada. En el contexto del consentimiento informado nunca puede o no debe infravalorarse, ya que el receptor obtiene información de vital importancia, como indicaciones, efectos secundarios, procedimientos, síntomas, tratamientos disponibles, riesgos, beneficios, etc. Comunicar es informar o, en palabras de Montalt i Resurrecció \& García Izquierdo (2016: 82), "informar es un acto de comunicación".

Asegurar la comprensión en ambos modos (oral y escrito) también forma parte del acto comunicativo. No podemos continuar sin mencionar la tendencia de la que partimos: la traducción centrada en el usuario (user-centered translation, UTC) orientada hacia el contexto y los participantes (context-oriented and participant-oriented research) de acuerdo con la clasificación de Soujanen et al. (2015). Conviene matizar que hablamos de traducción del formulario del consentimiento informado por ser el objeto del presente estudio, pero extrapolamos dicha materia a la interpretación, ya que el procedimiento de comunicar el consentimiento informado se realiza de modo oral cuando el contexto es 
multilingüe y no existe un formulario en la lengua origen del paciente, por lo que el intérprete tiene que intervenir.

Desde 1980, la tendencia en traducción e interpretación se ha orientado hacia la teoría funcional centrada en el propósito de la traducción. Sin embargo, Nord (2012: 32) mencionó el vacío existente en la aplicación de la teoría a la parte práctica por la dificultad que conlleva:

Audience orientation has been a particularly sensitive aspect of functionalist theory and applications from the start. Critics have been asking how translators know what the audience expects of a translation. Indeed, it is easy to talk about the audience's expectations but much more difficult to obtain empirical proof of what audiences (for certain genres or in certain non-linguistic fields) really expect.

Dos de los aspectos fundamentales dentro de esta corriente aplicada a la interpretación médica y que nos sirven para poner de manifiesto la necesidad de conocer las expectativas de los usuarios en esta tendencia al paciente activo son la utilidad (usability) y la experiencia del usuario (user experience), siguiendo la descripción de los autores (Soujanen et al. 2015: 2):

[...] usability refers to the ease with which users can use a product to achieve their goals $[\ldots]$ according to their expectations and without obstacles or hindrances $[\ldots]$ user experience is a holistic concept which includes all the user's emotions, beliefs, preferences, perceptions, physical and psychological responses, behaviours and accomplishments (Soujanen et al. 2015: 13).

Relacionado con la utilidad, un concepto emergente es el de la intuición (intuition), definido también en Soujanen et al. (2015: 16) como "our familiarity with something in light of our earlier world of experience with it".

Resulta relevante mencionar la afirmación de los mismos autores por su pertenencia al contexto del objeto de studio: "Translators are the user's representatives, and as members of design teams, translators can help to create a full user experience" (Soujanen et al. 2015: 13).

\subsection{Textos médicos dirigidos a pacientes: el consentimiento informado}

El ámbito de la traducción y la redacción médica abarca distintos géneros textuales. No obstante, como ya hemos anunciado previamente, nos centraremos en el consentimiento informado enmarcado dentro del género de los textos médicos dirigidos a pacientes y familiares según la clasificación de Gil Alberdi (2009: 39).

Se trata de uno de los documentos más importantes en la investigación biosanitaria y, por consiguiente, en la traducción médica. Nos sumamos a la opinión de varios autores, que coinciden en que "el consentimiento informado 
es un ejemplo claro de que no siempre los textos para pacientes son todo lo inteligibles que se desearía que fuesen" (Valentini et al. 2013; Prieto Velasco 2014). Destacan las palabras de Valentini et al. (2013: 1), también recogidas en Prieto Velasco (2014: 32):

A large literature supports the notion that the language used is not comprehensible to most people. Subjects may not fully read [informed consent form] because it is too long, they do not understand it, and are confused by medical and legal terms.

Uno de los motivos, como indican Montalt i Resurrecció y González Davies (2007: 60), es que no se ha tenido en cuenta el bagaje previo del destinatario prototípico al que se dirige dicho género a la hora de redactar los textos. En esta línea, en palabras de Villamañán et al. (2016: 210) "obtener el consentimiento informado implica que previamente se ha proporcionado información adaptada a su nivel de comprensión". Sin embargo, ¿cómo se adapta la información a dicho nivel de comprensión?

De este modo, podríamos afirmar que se observan diferencias en el plano conceptual y en el lingüístico que nos conducen a preguntas de investigación como: ¿qué rol emplea el intérprete en este contexto?, ¿cómo se combina la modalidad oral con la escrita? o ¿qué impacto pueden crear las barreras del lenguaje en términos de comprensibilidad?

Tras nuestras indagaciones, entre los posibles autores de los formularios de consentimientos informados se encuentran los médicos o enfermeros, personal de administración de hospitales, empresas farmacéuticas, empresas de suministros médicos, organizaciones de salud, organismos gubernamentales y empresas aseguradoras. Dada la diversidad de autores, elementos como el tenor, el estilo, el vocabulario y los matices varían en cada documento. Se crean para un público generalista sin aplicar patrones sobre edad, sexo, nivel intelectual, etc. y este hecho conlleva dificultades de comprensión, dado que el público receptor suele presentar características muy diversas y necesidades muy particulares.

Como recoge Gallego Borghini (2015: 17), en 1999 Ordovás et al. ya llegaron a la conclusión de que "para entender bien el $97 \%$ de los consentimientos informados es preciso contar con estudios medios o superiores", dato que nos refiere a la idea anterior de inteligibilidad y complejidad de comprensión del consentimiento informado.

El presente trabajo se adscribe a una línea de investigación relativa a la percepción que tienen los pacientes de la calidad de la comunicación. En este contexto destacan los estudios recientes de Longacre et al. (2015) y Mazzi et 
al. (2016) también reseñados en Montalt i Resurrecció \& García Izquierdo (2016: 82).

\section{La comprensibilidad como factor indispensable}

Algunos procedimientos, como es el caso del consentimiento informado, se emplean para transmitir información especializada. La explicación de esta información por parte del profesional sanitario se concibe como la vía para hacerla accesible a los destinatarios que compartan la lengua del emisor, pero que no posean el mismo nivel de conocimiento sobre el tema.

En este acto de transmisión y accesibilidad, uno de los aspectos que ha suscitado gran preocupación desde el punto de vista médico, ético y lingüístico es el problema de la comprensibilidad. En nuestro enfoque la abordamos a partir de la legibilidad textual (readability) y la percepción de los usuarios que acuden a las consultas médicas. La legibilidad textual, como bien desarrolla Prieto Velasco (2014: 31) en su definición, que casa con nuestro contexto de estudio, "debe entenderse como el conjunto de rasgos lingüísticos que facilitan la lectura del texto". Es necesario mencionar la legibilidad visual (legibility), que también tiene cabida en el estudio relacionada con el grado de dificultad o facilidad conceptual que tienen los receptores para entender el texto (Wolfer 2015; Prieto Velasco 2014).

Distintos autores han realizado propuestas para mejorar la comprensibilidad como las que destacamos a continuación: Meyer \& Mackintosh (2000) ahondan en la técnica de la desterminologización, entendida como el mecanismo para realizar cambios semánticos en los términos que pasan a emplearse en el lenguaje común; Mayor Serrano (2008) propone simplificar la estructura y realizar cambios a nivel microtextual y macrotextual; Muñoz Miquel (2012) recopiló los procedimientos de reformulación para mejorar la comprensión, como reestructurar el texto en su conjunto, seleccionar la información más relevante, incorporar elementos visuales, destacar las palabras clave, acotar las oraciones y simplificar estructuras sintácticas complejas, entre otros y Campos Andrés (2013), que analiza distintos recursos, como, por ejemplo, la definición, la sinonimia o la analogía para asegurar la eficacia comunicativa. Sin embargo, nuestra propuesta tiene un carácter novedoso debido a la introducción del intérprete como especialista en la gestión de la comunicación y experto en lengua y cultura para reformular y adaptar la información escrita de manera oral y en un formato entendible por el paciente. Dichas técnicas pueden ir desde la desterminologización, pasando por la empatía, hasta el uso de recursos audiovisuales. 


\section{Análisis empírico-analítico sobre comprensibilidad y comunicación}

\subsection{Materiales, métodos e informantes}

El punto de partida de este proyecto nace a partir de la publicación del Decálogo del consentimiento informado elaborado por la Comisión Central de Deontología de la Organización Médica Colegial (COEM 2016). El documento es de especial relevancia por las cifras que indica; entre ellas destacamos que el $70 \%$ de las reclamaciones sanitarias están relacionadas con las deficiencias en información y con el consentimiento informado.

El estudio realizado tiene como base material un corpus compilado ad hoc a partir de los resultados de distintas estrategias de búsqueda de repeticiones y concordancias con la herramienta AntConc. Finalmente, el corpus lo conforman seis consentimientos informados pertenecientes a la especialidad de odontología en español y sus traducciones al inglés; un total, por tanto, de 12 textos. Conviene matizar que se decidió que perteneciesen a la misma especialidad para que la muestra fuese homogénea y los resultados más exactos. Los informantes están divididos en tres grupos de acuerdo con la metodología y objetivos del estudio. Por un lado contamos con un primer grupo de odontólogos que llevan trabajando en la sanidad privada en España entre 12 y 15 años y tienen contacto con un volumen considerable de pacientes extranjeros. Por otro lado, formamos dos grupos de pacientes: uno de cinco pacientes españoles y otro de cinco pacientes británicos, todos ellos pertenecientes a la misma franja de edad (45-60 años) y formados cada uno por 3 mujeres y 2 varones por cuestiones de disponibilidad. La metodología empleada en nuestra investigación es doble: cualitativa, a través de varios grupos de discusión (focus groups), y cuantitativa a través de una encuesta para pacientes y otra para profesionales sanitarios. Los resultados de ambos métodos se complementan entre sí.

Se ha elaborado un total de tres grupos de discusión: odontólogos, pacientes españoles y pacientes británicos, de acuerdo con la división de los grupos de informantes. En la primera parte, centrada en los profesionales sanitarios, después del grupo de discusión han respondido individualmente a varias cuestiones semi-estructuradas extraídas del estudio de Valero Garcés (OFRIM 2011: 118-132) para comprobar su perspectiva de la calidad asistencial a pacientes extranjeros y el papel del intérprete. Así, en la segunda parte, centrada en los pacientes, se han realizado dos grupos de discusión (uno con pacientes británicos y otro con españoles) siguiendo el mismo esquema. A dichos grupos les ha seguido una fase de lectura de dos consentimientos informados que forman parte del corpus (uno sobre blanqueamiento dental y otro sobre implantología) y una encuesta para corroborar la percepción descrita en el grupo de discusión 
sobre el grado de comprensibilidad a partir de preguntas reformulatorias o de reexpresión en otras palabras. Para la elaboración de la encuesta para pacientes se ha empleado una escala LIKERT de cinco puntuaciones donde 1 equivale a totalmente de acuerdo y 5 a totalmente en desacuerdo. Los materiales para los pacientes británicos han sido las traducciones al inglés de los dos consentimientos empleados y la misma encuesta, pero en inglés. El motivo de trabajar con cada grupo de informantes en su lengua materna va ligado a nuestro objetivo de descubrir el grado de comprensión, primero en su lengua materna, para después analizar cómo el intérprete ayuda a su comprensibilidad cuando no se dispone de textos en la lengua del destinatario por diferir con la oficial del país en el que se encuentran.

\subsection{Análisis y resultados}

En el estudio dirigido a los odontólogos profesionales, en el grupo de discusión se trataron distintos temas que conciernen a la interpretación y el consentimiento informado. Por ejemplo, en cuanto a las preguntas sobre sus conocimientos y expectativas de una consulta interpretada, indican que tienen nociones sobre el trabajo del intérprete gracias al contacto diario que establecen, pero no se les ha preparado para trabajar juntos. Los formularios de consentimientos informados con los que trabajan a diario son generalistas, es decir, plantillas de colegios profesionales que se adaptan en términos meramente formales, como el logo, el número de colegiado o algún riesgo asociado que precise mención y no se encuentre en la plantilla. Normalmente, en la transmisión de la información especializada que aparece en el consentimiento informado otorgan prioridad a la parte escrita, ya que es la que queda reflejada, y la parte oral se centra en un comentario aclarador para responder a las dudas que el paciente tenga. Es decir, en contextos multilingües, el profesional sanitario entrega el formulario del consentimiento informado (modo escrito) y es el intérprete el que realiza una traducción a vista (modo oral). En este punto afloró la realidad actual en la consulta, aunque no se puede generalizar, dado que la muestra no es lo suficientemente grande; es cierto que relataban ocasiones puntuales donde el intérprete se ha quedado solo con el paciente, lo que da pie a que, si surgen dudas, se tenga que buscar al profesional para que vuelva a entrar a la consulta y explique todas las dudas. Como inconvenientes a esta práctica observamos la consiguiente ralentización del proceso de comprensión del consentimiento por parte del receptor, ya que se interrumpe el proceso mientras el profesional vuelve a entrar a la consulta. Reiteramos que es en ocasiones puntuales, dado que siempre que disponen de intérprete se sienten más aliviados por su confianza en que es un experto en lengua y 
cultura. Finalmente, por su percepción, los problemas de comprensión de los pacientes vienen dados por la dificultad que se les presenta, ya que no disponen de conocimientos amplios sobre esta materia en la mayoría de las ocasiones. Por otro lado, en la encuesta que respondieron los profesionales sanitarios, las cuestiones estaban relacionadas con su percepción sobre el papel del intérprete. Por ejemplo, un $100 \%$ coincidió en que el intérprete sanitario debe explicar trasfondos y significados de la consulta extranjera, además de aclarar tecnicismos a los usuarios. En otras funciones hubo más disparidad de resultados; por ejemplo, en si el intérprete debe o no aclarar afirmaciones imprecisas preguntando directamente a los usuarios, donde un $80 \%$ considera que sí debe hacerlo. Además, para tres de los encuestados, el intérprete debe omitir afirmaciones secundarias para evitar la pérdida de tiempo frente a dos que responden que no debe omitirlas. A tenor de los resultados se refleja que aún existe un vacío de conocimientos y unos límites difusos sobre el papel del intérprete en ciertos entornos, como es el sanitario.

El otro grupo de informantes es el de paciente. En el análisis de la legibilidad textual a través de una encuesta semi-estructurada, podemos mencionar el siguiente ejemplo extraído de la muestra estudiada con pacientes españoles:

1) Se preguntó a los pacientes participantes acerca de la comprensión de varios párrafos en su totalidad como: "raramente pueden producirse comunicaciones con los senos nasales o con las fosas nasales. Existe incluso la posibilidad de lesionar el seno maxilar y provocar una sinusitis". Los participantes respondieron estar en desacuerdo a excepción de uno de ellos, que marcó la opción de en total desacuerdo. Para corroborar el grado de dificultad, además de preguntas como la muestra anterior se confeccionó otro tipo de preguntas donde se dejaban unas líneas en blanco para que explicasen con sus propias palabras el significado del apartado anterior. Siguiendo el ejemplo anterior, tres de los participantes no aportaron ninguna idea con sus propias palabras ni intentaron reformular porque su comprensión del párrafo era muy baja, y dos intentaron aportar alguna idea, como "se relaciona con la nariz" o "algo que no se suele producir pero existe la posibilidad". Por tanto, con este tipo de respuestas se observa que el problema de la falta de comprensión lo ocasionan los términos especializados, como "comunicaciones" o "sinusitis".

2) Por otro lado, cuando se realiza el estudio con los pacientes británicos, también se corrobora que la idea general del párrafo se ha comprendido grosso modo. No obstante, el significado completo no se 
ha comprendido, dado que no son una audiencia experta. Por tanto, cuantas más facilidades se les ofrezcan, mayor será el grado de comprensión y reexpresión para tomar opiniones fundadas. Sin embargo, cabría matizar a qué parte corresponde el ofrecer facilidades, ya que por el momento no se contempla que sea el intérprete el que tenga dicha función, aunque en la actualidad ocurra en reiteradas ocasiones. Por ello, queda confirmado que el rol del intérprete sanitario en la consulta de odontología no queda delimitado. Tanto es así que la integración de la interpretación en el momento del consentimiento informado no se ha estudiado en profundidad, por lo que sus límites son difusos.

Para llegar a estas afirmaciones mostramos algunos datos relevantes, como el resultado sobre la comprensión del término filling en la encuesta para pacientes británicos. Todos los informantes han respondido agree a la comprensión del término, dado que no existe un sinónimo en un registro más elevado. Por tanto, al emplearse en el lenguaje general, la comprensión es total. Sin embargo, en la pregunta sobre el término peroxides en la frase "to prevent possible unwanted effects it is essential that you warn us of any allergy (particular to peroxides)", no es comprendido por los informantes, aunque, al disponer de contexto, 2 informantes se han decantado por undecided, 2 por disagree y 1 por strongly disagree. Es por ello que la balanza no se ha decantado hacia la incomprensión total, sino que, aunque no se comprende el término en sí, el contexto sirve de ayuda. A este análisis sobre la legibilidad textual podemos añadir otro ejemplo sobre la comprensión de "canine teeth whiten less than incisors". En esta ocasión, al disponer de contexto y no de un solo término aislado, la idea general sí es comprendida por todos los informantes, obteniendo como resultado 4 informantes undecided y 1 agree.

En cuanto al análisis y descubrimiento de las expectativas de los pacientes durante los grupos de discusión, estos indican que la figura que mejor puede realizar matices culturales en la consulta es el intérprete, por la preparación que se le presupone. Asimismo mencionan en el grupo de discusión que, si el profesional sanitario que les atiende es de otra nacionalidad o ha realizado estancias en el extranjero, puede que también sepa aclarar conflictos culturales. No obstante, en sus experiencias, siempre que un conflicto cultural ha surgido en sus consultas, el intérprete lo ha resuelto de manera satisfactoria y, cuando alguno de ellos se ha visto puntualmente envuelto en algún conflicto cultural menor y sin intérprete, ha encontrado dificultades para resolverlo aún sin ser un conflicto de gran transcendencia. 
Como hemos mencionado anteriormente, la legibilidad visual también tenía cabida en el estudio. En este sentido, los pacientes fueron preguntados sobre si la inclusión de imágenes en los formularios de consentimientos informados les mejora la comprensibilidad total. En su opinión, para facilitar la comprensión sí es un recurso de utilidad como herramienta aclaradora, sobre todo para la parte del procedimiento. Además de las imágenes, los modelos y los recursos audiovisuales proporcionados durante la consulta les han sido de gran ayuda cuando han podido disponer de ellos, aunque haya sido en contadas ocasiones. Es necesario matizar que son de gran ayuda sobre todo para el colectivo de pacientes británicos, que juegan con la dificultad añadida del acceso ocasional a documentos en su lengua materna.

Otros temas tratados en los grupos de discusión fueron el hábito de lectura de los consentimientos, el momento de la firma y la resolución de dudas. En el caso de pacientes extranjeros, los problemas de comprensión también están presentes, incluso en su propia lengua, pero en mayor medida en sus visitas médicas, donde los consentimientos informados solo se les entregan en español. Cuando esta última situación ocurre, disminuye totalmente el intento de la comprensión y el interés por la lectura de dichos documentos a sabiendas de su implicación legal.

\section{Conclusiones}

El cambio de modelo de asistencia sanitaria y la tendencia a la educación del paciente traen consigo cambios para los que se necesitan estudios concernientes a las nuevas prácticas necesarias en el ámbito de la interpretación sanitaria. El presente trabajo se concibe como una contribución a dicha línea de investigación. Conviene matizar que, aunque se haya incorporado en el corpus la traducción al inglés de los consentimientos informados, en la actualidad, en las consultas en el territorio español no siempre se ofrece el consentimiento en dicha lengua. Es decir, se suele entregar el consentimiento informado en español y se recurre al intérprete para garantizar la comprensión, dado que los profesionales sanitarios no siempre están dispuestos a entregar un consentimiento que no han supervisado por sus conocimientos lingüísticos.

Si bien el documento del consentimiento informado ha supuesto grandes avances en cuanto a empoderamiento del paciente, también reviste aspectos negativos, como la falta de comprensibilidad total por parte de los pacientes en el dialogo que se establece entre las partes. Es decir, existe una brecha entre la exposición de la información y su debido procesamiento por parte del receptor. Para que la comunicación llegue a buen puerto, la divulgación del conocimiento especializado debe ser eficaz, así como la atención que debe recibir el 
paciente como individuo y no como grupo o audiencia entendida como conjunto homogéneo. La clave en este contexto es la individualidad, el paciente como individuo con características y necesidades distintas de cualquier otro paciente, es decir, la necesidad, cada vez más imparable, de personalizar la comunicación. Es en este sentido donde se pone de manifiesto la necesidad de estudios y prácticas orientadas hacia la eficacia comunicativa que contemplen la gestión de la multimodalidad tratada anteriormente en el momento del traspaso de la información escrita de modo oral por parte del intérprete. Según nuestros resultados, como ventajas de dicha multimodalidad observamos que los pacientes extranjeros residentes en España están en posición de ventaja respecto a los pacientes autóctonos, dado que no tienen un intermediario como lo hace el intérprete con los pacientes extranjeros. En este sentido, es el intérprete el que suple las carencias de comprensión de los pacientes y pasa a formar parte de un proceso de mediación que debería propiciar el profesional sanitario tanto en contextos monolingües como bilingües.

Para categorizar el papel del intérprete en el contexto de la consulta médica, en la parte del consentimiento informado, afirmamos que está más en consonancia con el enfoque Patient Navigators (Crezee 2013), puesto que actúa como comunicador y resuelve conflictos culturales, pero sin tomar parte por el paciente, es decir, todos los enfoques de la abogacía han quedado atrás. La clave está en la comunicación; el intérprete ya no es un puente o conducto, como anteriores enfoques señalaban; el intérprete es un comunicador y, de los perfiles profesionales que participan en la consulta clínica, es el que mejor conoce las necesidades lingüísticas, culturales y comunicativas de los pacientes como receptores. Además, es el que conoce las claves para adaptarse al paciente y empoderar su grado de comprensibilidad. Sin embargo, el desconocimiento del perfil del intérprete por parte de los profesionales sanitarios desemboca en dificultades a la hora de trabajar en equipo. Es de suma importancia, pues, saber distinguir qué tareas son propias del intérprete y qué partes son trabajo extra, como puede ser el recabar el consentimiento de un paciente, aunque sí sea partícipe o colaborador del proceso.

No obstante, actividades como el grupo de discusión realizado contribuyen a la difusión y al paso del desconocimiento al conocimiento de sus funciones. En la práctica habitual, aspectos de la comunicación como la empatía, las emociones o la claridad se dan por sentado; así pues, actividades como seminarios, charlas o grupos de discusión hacen conscientes dichos aspectos, que quedan en un segundo plano para profesionales sanitarios y, por consiguiente, se invisibilizan, cuando no se pueden pasar por alto. 
La intención es, pues, aportar datos para conseguir una comunicación más adecuada basada en una mediación (interpretación, traducción, revisión, adaptación de textos...) que arroje un poco de luz al camino hacia el empoderamiento y bienestar del paciente y de todas las partes implicadas.

Tras los resultados obtenidos y analizados observamos que, en la práctica, los consentimientos se firman en su mayoría a ciegas en este contexto y, por lo general, el paciente se siente obligado a firmarlo, aunque no comprenda la totalidad del documento para que la intervención o tratamiento propuestos se lleven a cabo. Es decir, ocurre lo que denominamos a partir de este trabajo como 'consentimientos velados'; entiéndase el consentimiento como un instrumento de decisión del paciente. De este modo, el paciente firma como si tuviese un velo que no le permite ver con claridad el contenido, lo que en inglés se ha categorizado como blind consent (Plaut \& Bartlett 2012: 293-311). Esta situación no es aceptable en términos intralingüísticos e interlingüísticos, dado que no fomenta ni la comprensibilidad ni la adherencia a un tratamiento por parte de los receptores. Además, tampoco es aceptable en términos éticos.

El análisis de la percepción de pacientes ayuda a determinar el grado de satisfacción y a ofrecer datos concretos que se empleen con la intención de mejorar la comunicación entre profesionales sanitarios, pacientes e intérpretes. Para ello, todas las partes implicadas necesitan aunar sus esfuerzos hacia la educación del paciente y la mejora del trabajo en equipo. Pasar de informar a comunicar es un objetivo para cumplir a corto plazo. Por ello, algunos de los estudios que recientemente destacan en la línea de investigación a la que se adscribe el presente estudio y que persiguen la mejora de la documentación clínica, la atención al paciente y la accesibilidad a la información especializada son: Saiz Hontangas et al. (2016) y su estudio sobre las guías para pacientes o Toledo Chávarri et al. (2016), cuyo estudio se centra en los usos y necesidades de pacientes crónicos y el papel de la comunicación escrita. En suma, queda patente el aumento a un ritmo frenético de la necesidad de pasar del texto al contexto y la necesidad de una comunicación personalizada para garantizar la eficacia comunicativa.

\section{Referencias bibliográficas}

CAMBridge, Jan. (2002) "Interlocutor roles and the pressures on interpreters." En: Valero Garcés, Carmen \& Guzmán Mancho Barés (eds.) 2002. Traducción e Interpretación en los Servicios Públicos. Nuevas necesidades para nuevas realidades. Community interpreting and translating: new needs for new realities. Madrid: Servicio de Publicaciones de la Universidad de Alcalá, pp. 11-124. 
CAMPOS ANDRÉs, Olga. (2013) "Procedimientos de desterminologización: traducción y redacción de guías para pacientes." Panacea 14:37, pp. 48-52.

COEM. (2016) Decálogo del consentimiento informado. Versión electrónica: <http:// www.cgcom.es/noticias/2016/06/16_06_21_decalogo_consentimiento_informado>

CREZEE, Ineke. (2013) Introduction to healthcare for interpreters and translators. Amsterdam: John Benjamins Publishing Company.

EPSTEIN, Ronald; Peter Franks; Kevin Fiscella; Cleveland Shields; Sean Meldrum; Richard Kravitz \& Paul Duberstein. (2005) "Measuring patient-centered communication in patient-physician consultations." Theoretical and Practical issues. Social Science and Medicine 61, pp. 1516-1528.

GALLEGO BORGHINI, Lorenzo. (2015) La traducción inglés-español del consentimiento informado en investigación clínica. Barcelona: Fundación Dr. Antonio Esteve.

GARCÍA IZQUIERDO, Isabel \& Vicent Montalt i Resurrecció. (2013) "Equigeneric and Intergeneric Translation in Patient-Centred Care." Hermes Journal of Language and Communication in Business 51, pp. 39-51.

GIL-ALBERDi GONZÁLEZ, Beatriz. (2009) "El redactor de textos médicos como profesional independiente." En: Rico-VilladEMOROS, Fernando \& Vicente Alfaro (eds.) 2009. La redacción médica como profesión: Qué es y qué hace el redactor de textos médicos. Barcelona: Fundación Dr. Antonio Esteve.

LEANZA, Yvan. (2005) "Roles of community interpreters in pediatrics as seen by interpreters, physicians and researchers." Interpreting. International journal of research and practice in interpreting 7:2, pp. 167-192. Amsterdam: John Benjamins Publishing Company.

LONGACRE, Margaret; Thomas Galloway; Claudia Parvata \& Carolyn Fang. (2015) "Medical communication-related informational need and resource preferences among family caregivers for head and neck cancer patients." Journal of Cancer education 30:4, pp. 786-791.

MAYOR SERRANO, Blanca. (2005) "Análisis contrastivo (inglés-español) de la clase de texto folleto de salud e implicaciones didácticas para la formación de traductores médicos." Panacea 6:20, pp. 132-141.

Mayor Serrano, Blanca. (2008) Cómo elaborar folletos de salud destinados a los Pacientes. Barcelona: Fundación Dr. Antonio Esteve.

MAzzi, María Ángela et al. (2016) "How patients would like to improve medical consultations: insights from a multicentre European study." Patient Education and Counselling 99:1, pp. 51-60.

MEYER, Ingrid \& Kristen Mackintosh. (2000) "When terms move into our everyday lives: an overview of determinologization." Terminology 6:1, pp. 111-138.

MONTALT I RESURRECCIÓ, Vicent \& Isabel García Izquierdo. (2016) “¿Informar o comunicar? Algunos temas emergentes en comunicación para pacientes.” 
Panacea 27:44, pp. 81-84. Versión electrónica: <http://www.tremedica.org/ panacea/IndiceGeneral/n44_editorial.pdf>

Montalt i Resurrecció, Vicent \& María González Davies. (2007) Medical Translation Step by Step. Manchester: St Jerome.

MuÑoz MiQuel, Ana. (2012) "From the original article to the summary for patients:

Reformulation procedures in intralingual translation." Linguistica Antverpiensia, New Series Themes in Translation Studies 11, pp. 187-206. Versión electrónica: <http://repositori.uji.es/xmlui/bitstream/handle/10234/79290/56678. pdf? sequence $=1 \&$ is Allowed $=\mathrm{y}>$

MuÑoz Miquel, Ana. (2014) "El perfil y las competencias del traductor médico desde el punto de vista de los profesionales: una aproximación cualitativa." Trans 18, pp. 163-181. Versión electrónica: <http://www.trans.uma.es/ Trans_18/Trans18_163-181_art2.pdf>

NorD, Christiane. (2012) "Quo vadis, functional translatology?" Target 24:1, pp. 26-42.

OrdovÁs BAINES, Juan Pablo et al. (1999) "Análisis de las hojas de información al paciente para la obtención de su consentimiento informado en ensayos clínicos." Medicina Clínica 112:3, pp. 90-94.

Plaut, Victoria \& Robert Bartlett. (2012) "Blind consent? A social psychological investigation of non-readership of click-through agreements." Pubmed 36:4, pp. 293-311.

PRIETO Velasco, Juan Antonio. (2014) Representación gráfica de conceptos médicos: estudio de caso de la guía para pacientes Efectos secundarios del Taxol@ (paclitaxel). Universidad Jaume I, Castellón. Trabajo de fin de master.

SAIz HontAngas, Paula et al. (2016) "El uso de imágenes en guías para pacientes: una primera aproximación desde la perspectiva del nivel de activación del paciente." Panacea 17:44, pp. 99-110. Versión electrónica: <http://www. tremedica.org/panacea/IndiceGeneral/n44_tribuna-PSaiz-HontangasEtAl.pdf>

SÁnCHEZ MARTOS, Jesús. (2014) "La educación para la salud y las habilidades de comunicación ante los avances de la medicina personalizada." En: Sánchez Caro, Javier \& Fernando Abellán (eds.) 2014. Medicina personalizada. Aspectos científicos, bioéticos y jurídicos. Madrid: Fundación salud 2000, pp. 96-112.

Soujanen, Tytti; Kaisa Koskinen \& Tiina Touminen. (2015) User-centered translation. New York: Routlegde.

Toledo Chavarri, Ana et al. (2016) "El papel de la documentación escita en el empoderamiento en salud: un estudio cualitativo." Panacea 17:44, pp. 115122. Versión electrónica: <http://www.tremedica.org/panacea/IndiceGeneral/ n44_tribuna-AToledo-ChavarriEtAl.pdf>

VALENTINI, Miriam et al. (2013) "Application of a readability score in informed consent forms for clinical studies." Journal of Clinical Research and Bioethics 4:3, pp. 156-161. 
VALERO GARCÉS, Carmen. (2011) "Estudio para determinar el tipo y calidad de la comunicación lingüística con la población extranjera en los Centros de salud." En: El impacto de la inmigración en la población autóctona. Madrid: OFRIM, pp. 118-132.

VILLAMAÑÁn, Elena et al. (2016) "El consentimiento informado en investigación clínica, ¿entienden los pacientes lo que firman?" Farmacia hospitalaria 40:3, pp. 210-215. Versión electrónica: <http://dx.doi.org/10.7399/fh.2016.40.3.10411> WOLFER, Sascha. (2015) "Comprehension and comprehensibility." En: MAKSYMSKI, Karyn; Silke Gutermuth \& Silvia Hansen-Schirra (eds.) 2015. Translation and Comprehensibility: Arbeiten zur Theorie und Praxis des Übersetzens und Dolmetschens, vol. 72. Berlín: Frank \& Timme, pp. 22-52.

\section{NOTA BIOGRÁFICA / BIONOTE}

ELENA PÉREZ ESTEVAN es Graduada en Traducción e Interpretación (Universidad de Alicante), Máster en Comunicación Intercultural, Interpretación y Traducción para los Servicios Públicos (Universidad de Alcalá) y Máster en Comunicación médico-sanitaria (Universidad Jaume I). Es profesora del Departamento de Filología Moderna de la Universidad de Alcalá, institución en la que se ocupa de la docencia en las áreas de lengua inglesa y traducción especializada. Centra su actividad investigadora en las líneas de la formación e investigación en traducción e interpretación para los servicios públicos, la calidad en interpretación y las nuevas tecnologías. También es de destacar su labor profesional como intérprete médico en España y en Escocia para diversas instituciones sanitarias tanto públicas como privadas.

ELENA PÉREZ ESTEVAN holds a degree in Translation and Interpreting (University of Alicante), a master's degree in Intercultural communication, public service interpreting and translation (University of Alcalá) and a master's degree in medical and health translation (Jaume I University). Lecturer in English Language and Translation areas at the University of Alcalá, modern philology department. Her research interests include training in public services, interpreting quality and new technology. She has extensive professional experience working as a medical interpreter in different hospitals in Spain and Scotland for several public and private institutions. 
